\title{
Generalized conditions for coexistence of competing parasitoids on a shared host
}

\author{
Abhyudai Singh ${ }^{1}$
}

\begin{abstract}
Motivated by the univoltine life histories of insects residing in the temperate-regions of the world, there is a rich tradition of modeling arthropod host-parasitoid interactions using a discrete-time formalism. We introduce a general class of discrete-time models for capturing the population dynamics of two competing parasitoid species that attack the same vulnerable stage of the host species. These models are characterized by two density-dependent functions: an escape response defined by the fraction of hosts escaping parasitism; and a competition response defined by the fraction of parasitized hosts that develop into adult parasitoids of either species. Model analysis reveals remarkably simple stability conditions for the coexistence of competing parasitoids. More specifically, coexistence occurs, if and only if, the adult host density increases with host reproduction rate, and the log sensitivity of the competition response is less than half. The latter condition implies that any increase in the adult parasitoid density will result in a sufficiently slow increase in the fraction of parasitized hosts that develop into parasitoids of that type. We next consider a model motivated by differences in parasitism risk among individual hosts with risk from the two parasitoid species assumed to be independently distributed as per a Gamma distribution. In such models, the heterogeneity in host risk to each parasitoid is quantified by the corresponding Coefficient of Variation (CV). Our results show that parasitoid coexistence occurs for sufficiently large reproduction rate, if and only if, the sum of the inverse of the two $\mathrm{CV}$ squares is less than one. This result generalizes the " $\mathrm{CV}$ greater than one" rule that defined the stability for a single parasitoid-host system to a multi parasitoid-host community.
\end{abstract}

\section{INTRODUCTION}

The interaction between a single parasitoid species with its host is formulated as a discrete-time model

$$
\begin{aligned}
H_{t+1} & =R H_{t} f\left(H_{t}, P_{t}\right) \\
P_{t+1} & =R H_{t}\left[1-f\left(H_{t}, P_{t}\right)\right]
\end{aligned}
$$

where $H_{t}$ and $P_{t}$ are the adult host, and the adult parasitoid densities, respectively, at the start of year $t$ [1]-[3]. The model is motivated by a typical host/parasitoid life cycle as shown in Fig. 1, which consists of adult hosts emerging during spring, laying eggs that hatch into larvae [4]-[6]. Host larvae then overwinter in the pupal stage, and metamorphosize as adults the following year. Without loss of any generality, we assume that the host becomes vulnerable to parasitoid attacks in the larval stage. Adult female parasitoids emerge during spring, search and attack hosts by laying an egg into the body of the host.While adult parasitoids die after this time window, the parasitoid egg hatches into a juvenile parasitoid that grows at the host's expense by using it as a food source, and this ultimately results in the death of the host. The juvenile parasitoids pupate, overwinter, and emerge as adult parasitoids the following year. In (1), $R H_{t}$ is the host larval density exposed to parasitoid attacks at the start of the vulnerable stage, where $R>1$ denotes the number of viable eggs produced by each adult host. The function $f\left(H_{t}, P_{t}\right)<1$ is the fraction of host larvae escaping parasitism and is referred to as the escape response. Thus, $R H_{t} f\left(H_{t}, P_{t}\right)$ is the total larval density escaping parasitism to become adult hosts for next year. Finally, $R H_{t}\left[1-f\left(H_{t}, P_{t}\right)\right]$ is the density of parasitized larvae that give rise to adult (female) parasitoids in the next generation.

The simplest formulation of (1) is the classical Nicholson-Bailey model

$$
\begin{aligned}
H_{t+1} & =R H_{t} \exp \left(-c T P_{t}\right) \\
P_{t+1} & =k R H_{t}\left[1-\exp \left(-c T P_{t}\right)\right]
\end{aligned}
$$

with a parasitoid-dependent escape response $\exp \left(-c T P_{t}\right)$,where $c>0$ represents the rate at which parasitoids attack hosts, and $T$ is the duration of the host vulnerable stage [7]. The Nicholson-Bailey model is characterized by

\footnotetext{
${ }^{1}$ Abhyudai Singh is with the Departments of Electrical and Computer Engineering, Biomedical Engineering and Mathematical Sciences, University of Delaware, Newark, DE 19716, USA absingh@udel.edu
} 
diverging oscillations in population densities resulting in an unstable population dynamics [7]. Recent work has identified two orthogonal mechanisms by which stability can arise in models of the form (1):

- The first mechanism is when the escape response $f\left(P_{t}\right)$ only depends on the parasitoid density, and then the non-trivial host-parasitoid equilibrium is stable, if and only, if, the equilibrium adult host density is an increasing function of the host reproduction rate $R$ [8]. This type of stability arises through several related processes, such as, a fraction of the host population being in a refuge (i.e., protected from parasitoid attacks) [3], [9], large host-to-host difference in parasitism risk [8], [10]-[12], parasitoid interference [13]-[15], and aggregation in parasitoid attacks [16]-[18].

- The second mechanism is a Type III functional response where the parasitoid attack rate accelerates sufficiently rapidly with increasing host density [19]. Here the escape response $f$ depends on both the host and parasitoid density, and interestingly, in this case stability leads to the adult host equilibrium density being a decreasing function of the host reproduction rate $R$ [20].

A key focus of this work is to expand these results to multi-parasitoid communities. Towards that end, we consider two competing parasitoid species that attack the same vulnerable stage of the host species, as has been well documented in nature [21]-[27].

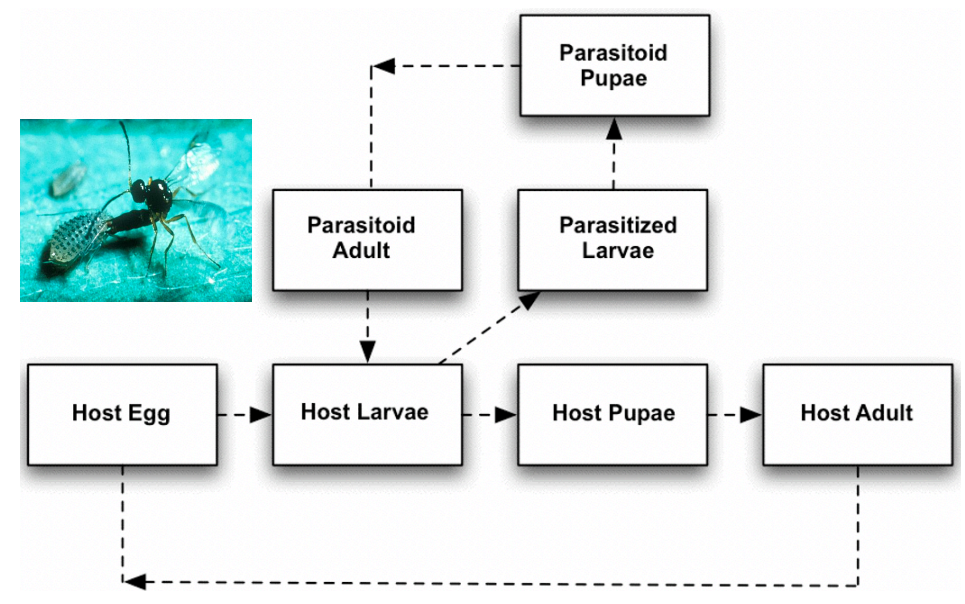

Fig. 1: Life cycle of the host and the parasitoid. Inset shows the picture of a parasitoid wasp laying an egg into the body of its host (spotted alfalfa aphid). Picture taken from https://en.wikipedia.org/wiki/Parasitoid.

\section{FORMULATION OF A PARASITOID-COMPETITION MODEL}

Following along the lines of model (1), we introduce a novel class of parasitoid-competition models that take the form

$$
\begin{aligned}
H_{t+1} & =R H_{t} f\left(P_{t}, Q_{t}\right) \\
P_{t+1} & =R H_{t}\left[1-f\left(P_{t}, Q_{t}\right)\right] g\left(P_{t}, Q_{t}\right) \\
Q_{t+1} & =R H_{t}\left[1-f\left(P_{t}, Q_{t}\right)\right]\left[1-g\left(P_{t}, Q_{t}\right)\right]
\end{aligned}
$$

where $P_{t}$ and $Q_{t}$ represent the densities of the two competing parasitoids in year $t$. The escape response $f\left(P_{t}, Q_{t}\right)$ is assumed to depend only on the parasitoid densities, and is a continuously differentiable function that is monotonically decreasing in both arguments. In the absence of both parasitoids

$$
f(0,0)=1,
$$

and the host population grows unboundedly as

$$
H_{t+1}=R H_{t}
$$


Recall that $R H_{t}\left[1-f\left(P_{t}, Q_{t}\right)\right]$ in the net density of parasitized larvae. The function $0 \leq g\left(P_{t}, Q_{t}\right) \leq 1$ represents the competition response that is the fraction of parasitized larvae that will develop into adult parasitoids $P_{t+1}$ in the next generation. Similarly, $1-g\left(P_{t}, Q_{t}\right)$ is the fraction of parasitized larvae that will develop into adult parasitoids $Q_{t+1}$. To be ecologically relevant, $g\left(P_{t}, Q_{t}\right)$ is an increasing function of $P_{t}$, but a decreasing function of $Q_{t}$ with the following properties

$$
g\left(0, Q_{t}\right)=0, \quad g\left(P_{t}, 0\right)=1 .
$$

Generally, a semi-discrete framework is needed for a derivation of (3) that mechanistically captures the population interactions during the host's vulnerable stage [?], [19], [28]-[31]. Taking a phenomenological approach, a simple example of the competition response is

$$
g\left(P_{t}, Q_{t}\right)=\frac{\left(c_{p} P_{t}\right)^{h}}{\left(c_{p} P_{t}\right)^{h}+\left(c_{q} Q_{t}\right)^{h}}
$$

for positive constants $c_{p}, c_{q}$ and $h \neq 1$.

The non-trivial equilibrium densities $H^{*}, P^{*}, Q^{*}$ of the competition model (3) satisfy

$$
\frac{1}{R}=f\left(P^{*}, Q^{*}\right), \quad P^{*}=(R-1) H^{*} g\left(P^{*}, Q^{*}\right), \quad \frac{P^{*}}{Q^{*}}=\frac{g\left(P^{*}, Q^{*}\right)}{1-g\left(P^{*}, Q^{*}\right)}
$$

where the last equation determines the ratio of the parasitoid densities. For example, the competition response (7) leads to the ratio

$$
\frac{P^{*}}{Q^{*}}=\left(\frac{c_{q}}{c_{p}}\right)^{\frac{h}{h-1}} .
$$

Before performing a systematic stability analysis, it is important to point out that model (3) generalizes previous multi parasitoid-host models, many of which implicitly assume that different parasitoid species attack different host developmental stages [32]-[36].

\section{COnditions For PARASitoid COEXISTENCE}

We begin by defining dimensionless log sensitivities of the equilibrium densities to the host reproduction rate $R$

$$
H_{R}:=\frac{R}{H^{*}} \frac{d H^{*}}{d R}, \quad P_{R}:=\frac{R}{P^{*}} \frac{d P^{*}}{d R}, \quad Q_{R}:=\frac{R}{Q^{*}} \frac{d Q^{*}}{d R} .
$$

Similarly, we defining log sensitivities of the escape/competition response to the parasitoid densities

$$
\begin{aligned}
& f_{P}:=\left.\frac{P^{*}}{f\left(P^{*}, Q^{*}\right)} \frac{\partial f\left(P_{t}, Q_{t}\right)}{\partial P_{t}}\right|_{P_{t}=P^{*}, Q_{t}=Q^{*}, \quad f_{Q}}:=\left.\frac{Q^{*}}{f\left(P^{*}, Q^{*}\right)} \frac{\partial f\left(P_{t}, Q_{t}\right)}{\partial Q_{t}}\right|_{P_{t}=P^{*}, Q_{t}=Q^{*},} \\
& g_{P}:=\left.\frac{P^{*}}{g\left(P^{*}, Q^{*}\right)} \frac{\partial g\left(P_{t}, Q_{t}\right)}{\partial P_{t}}\right|_{P_{t}=P^{*}, Q_{t}=Q^{*}, \quad g_{Q}}:=\left.\frac{Q^{*}}{g\left(P^{*}, Q^{*}\right)} \frac{\partial g\left(P_{t}, Q_{t}\right)}{\partial Q_{t}}\right|_{P_{t}=P^{*}, Q_{t}=Q^{*}},
\end{aligned}
$$

where $\left.\frac{\partial f\left(P_{t}, Q_{t}\right)}{\partial P_{t}}\right|_{P_{t}=P^{*}, Q_{t}=Q^{*}}$ represents the partial derivative of the escape response with respect to $P_{t}$ evaluated at the equilibrium point. Note that for the competition response (7) the $\log$ sensitivities sum to zero, i.e.,

$$
g_{P}=-g_{Q}
$$

irrespective of $c_{p}, c_{q}$ and $h$. Considering small perturbations $h_{t}, p_{t}$ and $q_{t}$

$$
h_{t}=H_{t}-H^{*}+h_{t}, \quad p_{t}=P_{t}-P^{*}, \quad q_{t}=Q_{t}-Q^{*},
$$

and linearizing model nonlinearities in (3) around the equilibrium, results in the following linear discrete-time system

$$
\left[\begin{array}{c}
h_{t+1} \\
p_{t+1} \\
q_{t+1}
\end{array}\right]=A\left[\begin{array}{l}
h_{t} \\
p_{t} \\
q_{t}
\end{array}\right], A=\left[\begin{array}{lll}
a_{11} & a_{12} & a_{13} \\
a_{11} & a_{12} & a_{13} \\
a_{11} & a_{12} & a_{13}
\end{array}\right]
$$


where the entries of the Jacobian matrix $A$ are given by

$$
\begin{aligned}
& a_{11}=1 \\
& a_{12}=\frac{H^{*} f_{P}}{P^{*}} \\
& a_{13}=\frac{H^{*} f_{Q}}{Q^{*}} \\
& a_{21}=(R-1) g\left(P^{*}, Q^{*}\right) \\
& a_{22}=g_{P}-\frac{f_{P}}{R-1} \\
& a_{23}=\frac{P^{*} g_{Q}}{Q^{*}}-\frac{P^{*} f_{Q}}{Q^{*}(R-1)} \\
& a_{31}=(R-1)\left(1-g\left(P^{*}, Q^{*}\right)\right) \\
& a_{32}=-g_{P}-\frac{Q^{*} f_{P}}{P^{*}(R-1)} \\
& a_{33}=-\frac{P^{*} g_{Q}}{Q^{*}}-\frac{f_{Q}}{(R-1)} .
\end{aligned}
$$

To derive analytical conditions for the stable coexistence of all three species we use the following result. Let

$$
\begin{aligned}
& c_{1}=-a_{11}-a_{22}-a_{33} \\
& c_{2}=\sum_{k=1}^{3} \text { Det } A_{k} \\
& c_{3}=- \text { Det } A
\end{aligned}
$$

where $A_{k}$ is the $2 \times 2$ matrix obtained from matrix $A$ by deleting row $k$ and column $k$, and Det represents the matrix determinant. Then, the non-trivial fixed point $H^{*}, P^{*}, Q^{*}$ is asymptotically stable, if and only if, the following inequalities hold

$$
\begin{aligned}
& 1+c_{1}+c_{2}+c_{3}>0 \\
& 1-c_{1}+c_{2}-c_{3}>0 \\
& \left|c_{2}-c_{1} c_{3}\right|<1-c_{3}^{2}
\end{aligned}
$$

[37], [38].

\section{A. The symmetric case}

Consider the symmetric case with equal densities $P^{*}=Q^{*}$ and assuming (13), our analysis shows that inequality (18a) holds iff

$$
g_{P}<\frac{1}{2}
$$

Inequality (18b) is always true and inequality (18c) holds iff

$$
\frac{d H^{*}}{d R}>0
$$

Thus, competing parasitoids can coexist as long as the adult host density is an increasing function of $R$, and a sufficiently low sensitivity of the competition response to the parasitoid density. For the symmetric case $c=c_{p}=c_{q}$, $P^{*}=Q^{*}, g_{P}<\frac{1}{2}$ corresponds to having $h<1$ in (7). While these results hold for any general function $f$, we illustrate them using the escape response

$$
f\left(P_{t}, Q_{t}\right)=\frac{1}{\left(1+c_{p} C V_{p}^{2} P_{t}\right)^{\frac{1}{C V_{p}^{2}}}} \frac{1}{\left(1+c_{q} C V_{q}^{2} Q_{t}\right)^{\frac{1}{C V_{q}^{2}}}}
$$


that is motivated by differences in parasitism risk among individual hosts. In particular, for each host, the parasitoids have a different attack rate (i.e., risk) that is assumed to be independent between the two species and follows a Gamma distribution [8], [12]. Here, $c_{p}$ and $c_{q}$ denote the average attack rates for parasitoids $P$ and $Q$ with the coefficient of variation in the attack rate given by $C V_{p}$ and $C V_{q}$, respectively. An alternative interpretation of this escape response is that the parasitoid species independently aggregate attacks on a subpopulation of hosts with $1 / C V_{p}$ and $1 / C V_{q}$ quantifying the extent of clumping in parasitoid attacks [33]. For the symmetric case $c=c_{p}=c_{q}$, $C V=C V_{p}=C V_{q}$

$$
f\left(P_{t}, Q_{t}\right)=\frac{1}{\left(1+c C V^{2} P_{t}\right)^{\frac{1}{C V^{2}}}} \frac{1}{\left(1+c C V^{2} Q_{t}\right)^{\frac{1}{C V^{2}}}}
$$

which solving (8) leads to the adult host equilibrium density

$$
H^{*}=\frac{2\left(R^{\frac{C V^{2}}{2}}-1\right)}{c(R-1)}
$$

that is an increasing function of $R$ (and hence, a stable equilibrium) iff $C V^{2}>2$. In essence, coexistence of symmetric parasitoids on their shared host requires $g_{P}<\frac{1}{2}$ and $C V^{2}>2$. Note that stable interaction of a single-parasitoid species and its host requires $C V^{2}>1$ [8], [10]-[12], and our result $C V^{2}>2$ shows that coexistence of multiparasitoid communities requires hosts to have much larger variation in parasitism risk. These results are illustrated in Fig. 2 and 3 where a low sensitivity of the competition response $(h<1$ in (7)) leads to coexistence of all three species. In contrast, a high sensitivity of the competition response $(h>1$ in (7)) leads to extinction of one of the parasitoid species. It is interesting to point out that coexistence of competing parasitoids leads to a much lower host density in Fig. 2 as compared to a single parasitoid species in Fig. 3, and this has important implications for biological control of pests [39]-[42].
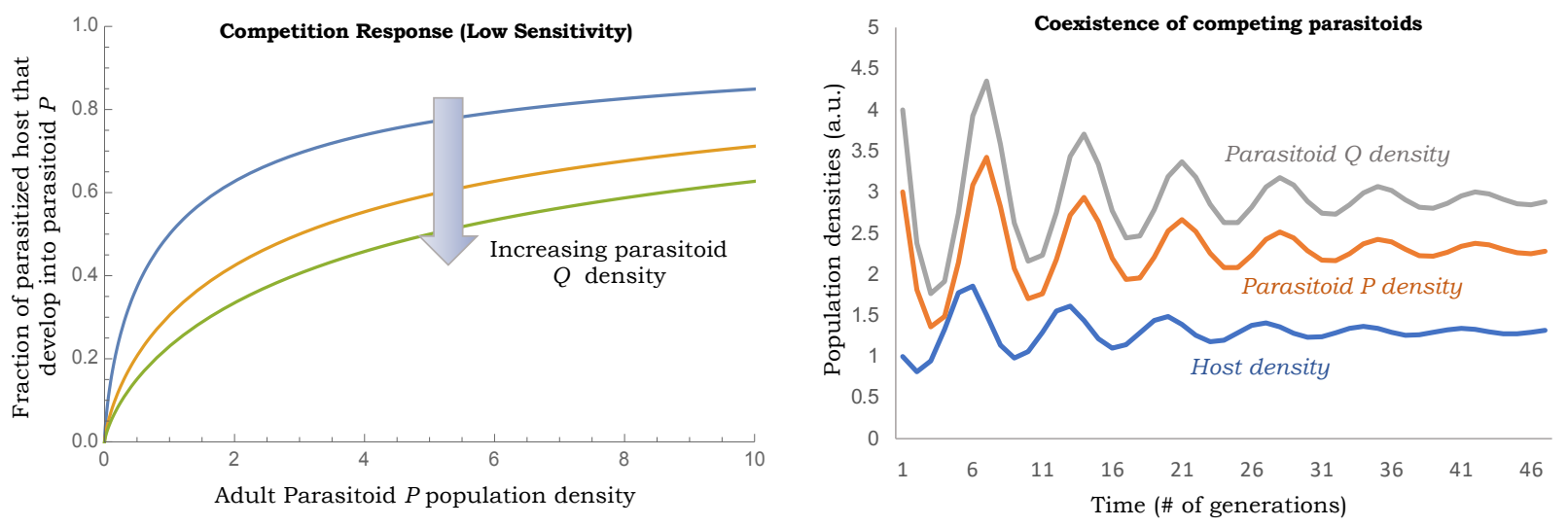

Fig. 2: Low sensitivity of the competition response leads to coexistence of competing parasitoids together with their shared host. Left: Competition response (7) plotted as a function of parasitoid $P$ population density for $h=0.7$ and $c_{p}=c_{q}=1$. Right: Simulation of model (3) with escape response (21) and competition response (7) for $c_{p}=0.95, c_{q}=1.05, C V=C V_{p}=C V_{q}=2.5$. To contrast the population time series, parasitoid $Q$ is assumed to have a slightly higher attack rate than parasitoid $P$.

\section{B. The asymmetric case}

We next turn our attention to the asymmetric case where $g\left(P^{*}, Q^{*}\right) \neq 1 / 2$ that results in

$$
\frac{P^{*}}{Q^{*}}=\frac{g\left(P^{*}, Q^{*}\right)}{1-g\left(P^{*}, Q^{*}\right)} \neq 1 \text {. }
$$



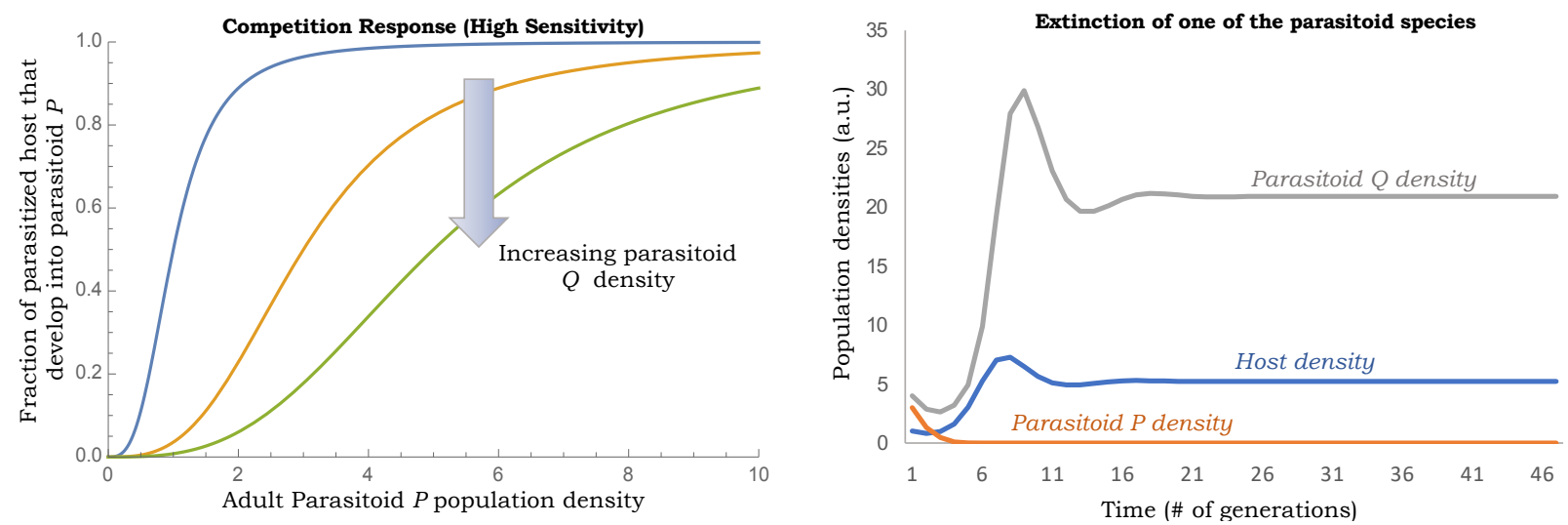

Fig. 3: High sensitivity of the competition response leads to extinction of one the parasitoid species. Left: Competition response (7) plotted as a function of parasitoid $P$ population density for $h=1.3$ and $c_{p}=c_{q}=1$. Right: Simulation of model (3) with escape response (21) and competition response (7) for $c_{p}=0.95, c_{q}=1.05$, $C V=C V_{p}=C V_{q}=2.5$. The lower attack rate of parasitoid $P$ results in its extinction, in spite of having $C V>2$.

It turns out that assuming (13), inequality (18a) becomes

$$
1+c_{1}+c_{2}+c_{3}=\frac{1-g\left(P^{*}, Q^{*}\right)-g_{P}}{1-g\left(P^{*}, Q^{*}\right)} \frac{R-1}{R+(R-1) H_{R}}>0
$$

resulting in the stability condition

$$
g_{p}<1-g\left(P^{*}, Q^{*}\right)
$$

together with (20) that is still needed to satisfy inequality (18c). A simple interpretation of (26) is that as one parasitoid species gets more dominant, coexistence requires the competition response to become even more insensitive. Revisiting the escape response (21) and assuming $R \gg 1$ such that

$$
f\left(P_{t}, Q_{t}\right) \approx \frac{1}{\left(c_{p} C V_{p}^{2} P_{t}\right)^{\frac{1}{C V_{p}^{2}}}} \frac{1}{\left(c_{q} C V_{q}^{2} Q_{t}\right)^{\frac{1}{C V_{q}^{2}}}}
$$

results in

$$
H^{*}=\frac{\left(R^{\frac{1}{\frac{1}{C V_{p}^{2}}+\frac{1}{C V_{q}^{2}}}}-1\right)}{g\left(P^{*}, Q^{*}\right)(R-1)\left(c_{p} C V_{p}^{2}\right)^{\frac{1}{C V_{p}^{2}}}\left(c_{q} C V_{q}^{2} \frac{1-g\left(P^{*}, Q^{*}\right)}{g\left(P^{*}, Q^{*}\right)}\right)^{\frac{1}{C V_{q}^{2}}}} .
$$

The adult host density being an increasing function of $R$ leads to the stability criterion

$$
\frac{1}{C V_{p}^{2}}+\frac{1}{C V_{q}^{2}}<1
$$

For the symmetric case $C V=C V_{p}=C V_{q}$, (29) reduces to our earlier result $C V^{2}>2$, and also implies that having both $C V_{p}^{2}, C V_{q}^{2}>1$ is a necessary condition for coexistence.

In summary, we have introduced a class of discrete-time models that captures competition of parasitoids on a shared host. The necessary and sufficient conditions for coexistence of all three species turns to be simple and elegant: the adult host equilibrium increases with the host reproduction rate and the inter-species competition is sufficiently small. The latter condition is captured by the log-sensitivity of the competition response being low as given by (26) where any increase in the parasitoid $P$ density does not lead to a sharp decrease in the parasitized larvae for parasitoid $Q$. Our stability results can easily be tested with field observation by monitoring population 
densities across generations. Future work will focus on incorporating correlations in the attack rate, allowing for one of the parasitoid to have a Type III functional response (which can also be strongly stabilizing [19]), and also exploring spatial mechanism for parasitoid coexistence [43]-[46].

\section{REFERENCES}

[1] M. P. Hassell. New York: Oxford University Press, 2000.

[2] W. S. C. Gurney and R. M. Nisbet, Ecological Dynamics. Oxford University Press, 1998.

[3] W. W. Murdoch, C. J. Briggs, and R. M. Nisbet, Consumer-Resouse Dynamics. Princeton,NJ: Princeton University Press, 2003.

[4] A. E. Hajek, Insect parasitoids: attack by aliens. Cambridge University Press, 2004, p. 145169.

[5] H. C. J. Godfray, Parasitoids; Behavioral and Evolutionary Ecology. 41 William St, Princeton, NJ 08540: Princeton University Press, 1994.

[6] J. Waage and D. Greathead, Insect Parasitoids. Academic Press, 1986.

[7] A. Nicholson and V. A. Bailey, "The balance of animal populations. part 1." Proc. of Zoological Society of London, vol. 3, pp. 551-598, 1935.

[8] A. Singh, W. W. Murdoch, and R. M. Nisbet, "Skewed attacks, stability, and host suppression," Ecology, vol. 90, no. 6, pp. 1679-1686, 2009.

[9] E. Bešo, S. Kalabušić, N. Mujić, and E. Pilav, "Stability of a certain class of a host-parasitoid models with a spatial refuge effect," Journal of Biological Dynamics, vol. 14, no. 1, pp. 1-31, 2020.

[10] A. D. Taylor, "Heterogeneity in host-parasitoid interactions: 'aggregation of risk' and the ' $c v^{2}>1$ rule.'," Trends in Ecology and Evolution, vol. 8, pp. 400-405, 1993.

[11] M. P. Hassell, R. M. May, S. W. Pacala, and P. L. Chesson., "The persistence of host-parasitoid associations in patchy environments. I. a general criterion." American Naturalist, vol. 138, pp. 568-583, 1991.

[12] S. W. Pacala and M. P. Hassell., "The persistence of host- parasitoid associations in patchy environments. II. evaluation of field data." American Naturalist, vol. 138, pp. 584-605, 1991.

[13] C. Bernstein, "Density dependence and the stability of host-parasitoid systems," Oikos, pp. 176-180, 1986.

[14] C. Free, J. Beddington, and J. Lawton, "On the inadequacy of simple models of mutual interference for parasitism and predation," The Journal of Animal Ecology, pp. 543-554, 1977.

[15] D. Rogers and M. Hassell, "General models for insect parasite and predator searching behaviour: interference," The Journal of Animal Ecology, pp. 239-253, 1974.

[16] J. D. Reeve, J. T. Cronin, and D. R. Strong., "Parasitoid aggregation and the stabilization of a salt marsh host- parasitoid system," Ecology, vol. 75, pp. 288-295, 1994.

[17] P. Rohani, H. C. J. Godfray, and M. P. Hassell, "Aggregation and the dynamics of host-parasitoid systems: A discrete-generation model with within-generation redistribution," The American Naturalist, vol. 144, no. 3, pp. 491-509, 1994.

[18] R. M. May, "Host-parasitoid systems in patchy environments: a phenomenological model," Journal of Animal Ecology, vol. 47, pp. 833-844, 1978.

[19] A. Singh and R. M. Nisbet, "Semi-discrete host-parasitoid models," Journal of Theoretical Biology, vol. 247, no. 4, pp. 733-742, 2007.

[20] A. Singh and B. Emerick, "Hybrid systems modeling of ecological population dynamics," bioRxiv, 2020.

[21] C. E. Bográn, K. M. Heinz, and M. A. Ciomperlik, "Interspecific competition among insect parasitoids: field experiments with whiteflies as hosts in cotton," Ecology, vol. 83, no. 3, pp. 653-668, 2002.

[22] W. Hogarth and P. Diamond, "Interspecific competition in larvae between entomophagous parasitoids," The American Naturalist, vol. 124, no. 4, pp. 552-560, 1984.

[23] S. Ortiz-Martínez, J.-S. Pierre, J. van Baaren, C. Le Lann, F. Zepeda-Paulo, and B. Lavandero, "Interspecific competition among aphid parasitoids: molecular approaches reveal preferential exploitation of parasitized hosts," Scientific reports, vol. 9, no. 1, pp. 1-11, 2019.

[24] Y. Feng, S. Wratten, H. Sandhu, and M. Keller, "Interspecific competition between two generalist parasitoids that attack the leafroller epiphyas postvittana (lepidoptera: Tortricidae)," Bulletin of entomological research, vol. 105, no. 4, pp. 426-433, 2015.

[25] H.-Y. Xu, N.-W. Yang, and F.-H. Wan, "Competitive interactions between parasitoids provide new insight into host suppression," PloS one, vol. 8, no. 11, p. e82003, 2013.

[26] J. A. Harvey, E. H. Poelman, and T. Tanaka, "Intrinsic inter-and intraspecific competition in parasitoid wasps," Annual Review of Entomology, vol. 58, pp. 333-351, 2013

[27] M. B. Bonsall, M. P. Hassell, P. M. Reader, and T. H. Jones, "Coexistence of natural enemies in a multitrophic host-parasitoid system," Ecological Entomology, vol. 29, no. 6, pp. 639-647, 2004.

[28] A. Singh and R. M. Nisbet, "Variation in risk in single-species discrete-time models," Mathematical Biosciences and Engineering, vol. 5, pp. 859-875, 2008.

[29] B. K. Emerick and A. Singh, "The effects of host-feeding on stability of discrete-time host-parasitoid population dynamic models." Mathematical Biosciences, vol. 272, pp. 54-63, 2016.

[30] E. Pachepsky, R. M. Nisbet, and W. W. Murdoch, "Between discrete and continuous: Consumer-resource dynamics with synchronized reproduction," Ecology, vol. 89, no. 1, pp. 280-288, 2007.

[31] B. K. Emerick and A. Singh, "Global redistribution and local migration in semi-discrete host-parasitoid population dynamic models." Mathematical Biosciences, vol. 327, p. 108409, 2020.

[32] N. Kakehashi, Y. Suzuki, and Y. Iwasa, "Niche overlap of parasitoids in host-parasitoid systems: its consequence to single versus multiple introduction controversy in biological control," Journal of Applied Ecology, pp. 115-131, 1984.

[33] R. M. May and M. P. Hassell, "The dynamics of multiparasitoid-host interactions," The American Naturalist, vol. 117, no. 3, pp. 234-261, 1981.

[34] C. Briggs, R. Nisbet, and W. Murdoch, "Coexistence of competing parasitoid species on a host with a variable life cycle," Theoretical Population Biology, vol. 44, no. 3, pp. 341-373, 1993.

[35] E. Hackett-Jones, C. Cobbold, and A. White, "Coexistence of multiple parasitoids on a single host due to differences in parasitoid phenology,” Theoretical Ecology, vol. 2, no. 1, pp. 19-31, 2009. 
[36] E. van Velzen, S. Pérez-Vila, and R. S. Etienne, "The role of within-host competition for coexistence in multiparasitoid-host systems," The American Naturalist, vol. 187, no. 1, pp. 48-59, 2016.

[37] G. Ledder, Mathematics for the life sciences: calculus, modeling, probability, and dynamical systems. Springer Science \& Business Media, 2013.

[38] S. Elaydi, An Introduction to Difference Equations. Newyork: Springer, 1996.

[39] S. D. Lane, C. M. St. Mary, and W. M. Getz, "Coexistence of attack-limited parasitoids sequentially exploiting the same resource and its implications for biological control," in Annales Zoologici Fennici. JSTOR, 2006, pp. 17-34.

[40] B. S. Pedersen and N. J. Mills, "Single vs. multiple introduction in biological control: the roles of parasitoid efficiency, antagonism and niche overlap," Journal of Applied Ecology, vol. 41, no. 5, pp. 973-984, 2004.

[41] P. K. Abram, J. Brodeur, V. Burte, and G. Boivin, "Parasitoid-induced host egg abortion; an underappreciated component of biological control services provided by egg parasitoids." Biological Control, no. 98, pp. 52-60, 2016.

[42] M. A. Jervis, B. A. Hawkin, and N. A. C. Kidd, "The usefulness of destructive host-feeding parasitoids in classical biological control: theory and observation conflict," Ecological Entomology, vol. 21, no. 1, pp. 41-46, 1996.

[43] D. Slone and J. Allen, "Persistence of multiple identical parasitoid species in a single-host, spatial simulation," Web Ecology, vol. 5, no. 1, pp. 6-13, 2005.

[44] E. E. Porter and B. A. Hawkins, "The influence of varying spatial heterogeneity on the refuge model for coexistence of specialist parasitoid assemblages," Oikos, vol. 100, no. 2, pp. 241-250, 2003.

[45] R. E. Snyder, E. T. Borer, and P. Chesson, "Examining the relative importance of spatial and nonspatial coexistence mechanisms," The American Naturalist, vol. 166, no. 4, pp. E75-E94, 2005.

[46] P. Amarasekare, "Competitive coexistence in spatially structured environments: a synthesis," Ecology letters, vol. 6, no. 12, pp. 1109-1122, 2003. 